\title{
Prevalence of Suicide Thoughts, Plans and Attempts in a Community Sample from Karaj, Iran
}

\author{
Seyed Kazem Malakouti ; Marzieh Nojomi ; Jafar Bolhari ; Mitra Hakimshooshtari ; Marjan Poshtmashhadi ; Diego De Leo
}

Abstract Aim of this study was to evaluate the prevalence of suicide ideation and behavior in a community sample of an Iranian city, Karaj. Study sample consisted of 2,300 subjects, chosen randomly from the community. The WHO/SUPRE-MISS survey questionnaire, used in this study. Lifetime prevalence for suicide thoughts, plans and attempts were 12.7, 6.2 and 3.3\%, respectively and for the previous year they were 5.7, 2.9 and 1\%, respectively. Although it is not possible to generalize the results to the country, but in some area of Iran, the prevalence of suicide ideation, plan and attempt appeared to be similar to that of many developed countries.

Keywords Suicide ideation _ Suicide attempt_Community survey _ Epidemiology _ Iran

Introduction

Suicide prevention is one of the World Health Organization priorities in mental health also for developing countries. Approximately 1 million people die by suicide every year worldwide, with China and India contributing nearly half of this number WHO/EURO (2005).

So far, rates of suicide in Iran have been drawn from studies performed in different settings, with the most recent available data originating from the Iranian Legal Medicine Organization (ILMO). According to this source, nationwide rates of suicide were 5.7 per 100,000 for males and 3.1 for females (Moradi and Khademi 2002). The highest figures belonged to the western provinces of the country, with rates ranging in some cities between 40 and 66 cases per 100,000 (Moradi and Khademi 2002).

Little information is available about suicide ideation, plans and attempts from community investigations of Iranian populations. The only example of such studies is the community survey performed in the context of the WHO/ SUPRE-MISS project in Karaj $(n=504)$, which evidenced that lifetime suicide ideation was present in $14 \%$ of subjects, while it was recorded in $6 \%$ of cases in the year prior to the investigation (Bertolote et al. 2005).

Internationally, studies from different cultural environments revealed that the prevalence of suicide ideation for lifetime, previous 12, 6 and 1 month, was 10.5-13.5\% (community samples from Australia and the US), 8.6-12\% (national surveys in Sweden in 1986 and 1996), 19.3\% (students with age range of 12-18 years in rural areas of one province in China) and 6.3\% (community sample from Casablanca, Morocco) (Agoub et al. 2006; De Leo et al. 2005; Kessler et al. 1999; Renberg 2001).

In the context of suicide plans, the study of Rey et al. (1997) indicated that, in the year preceding the investigation, the planning of suicide was $14.7 \%$, whilst it was just 4.4\% (lifetime) in an Australian community study (De Leo et al. 2005). Results from the WHO/SUPRE-MISS study indicated that in Iran suicide planning was present in 3\% of cases for the year before the interview and 6.6\% lifetime (Bertolote et al. 2005).

Studies of suicide attempts in the community have produced results ranging from 0.2 to $2.6 \%$ (previous year) and 2.6 to $10 \%$ (lifetime) (De Leo et al. 2005; Kessler et al. 1999; Liu et al. 2005; Renberg 2001).

The scarcity of studies carried out thusfar on suicidal behaviors in Iran and Middle East countries may be attributed to cultural barriers, the presence of a strong stigma on mental disorders (particularly on suicide), and the lack of registration systems. Given that suicidal behaviors are influenced by several medical, social, cultural, economical and political factors, understanding the differences between western and eastern societies by conducting epidemiological studies in this region of the world could be considered a necessary exercise towards advancing cross-cultural comparisons.

This study is the first to have been carried out in Iran as a developing country by using a community sample of considerable size. 
This study was conducted in Karaj during April-August 2005. About 2,300 subjects were recruited for the present research. Participants were randomly selected from a list of 305,000 families, sorted according to the electric power company codes of each house. From every family, one available person over the age of 15 years was randomly selected. Before filling in the questionnaires, confidentiality of personal data was emphasized. Subjects signed an informed consent that was approved by the Iranian National Committee for Research on Medical Sciences. Interviews were conducted by ten trained operators with a BS degree in psychology.

\section{Measures}

The instrument adopted was the questionnaire used in the WHO/SUPRE-MISS community survey (Fleischmann et al. 2005), translated in Farsi by psychiatrists and clinical psychologists, and back-translated for accuracy at the time of its first implementation. Suicidal behaviors were evaluated using the following key-questions: "Have you ever seriously thought of putting an end to your life?’, “Have you ever made a plan to do this?”, and “Have you ever attempted suicide?”. Additional information was collected in cases where the interviewee gave an ffrmative answer to any of these questions. SPSS 11.5 was used for all statistical analysis, with the level of significance set at $\mathrm{P} \backslash 0.05$.

\section{Results}

The demographic characteristics of the study sample are shown in Table 1. Most subjects were females (64.8\%), married (57.2\%), of high school educational level (48\%) and 45.4\% were housewives. Most subjects were in the age range of 20-24 years (20.7\%). There was no significant correlation between marital status and suicide attempt in female subjects. However, $51.9 \%(n=27)$ of the married females had a history of suicide attempts compared to $40.4 \%(n=21)$ of females who were single. Similar evidence was also found by Zakiullah et al. (2008) in Pakistan, and is probably related to the fact that marriages—as is the case for Iran-are arranged by familial or social obligations. In males, the status of single showed a significant correlation with suicide attempts: $29.2 \%(n=7)$ of male with history of suicide attempt(s) were married compared to $62.5 \%(n=15)$ of the single males $(v=20.69$, $\mathrm{P} \backslash 0.001)$. More demographic correlations and risk factors are discussed in another article (Nojomi et al. 2007).

Results pertaining to the distribution of suicide ideation and behavior are seen in Table 2. Phenomena recorded during lifetime span resulted approximately three times more frequently than those reported in the 12-month period preceding the interview.

\section{Discussion}

This is the first study on suicide ideation and behavior conducted in Iran on a rather large community sample. With the exception of a few studies using fofial data from the Primary Health Care registration system of the Ministry of Health, or with door-to-door surveys published nationally (Bolhari et al. 2002; Moosavi et al. 2000; Naghavi and Akabari 2002), almost all the other studies were performed in clinical settings and emergency departments, or were based on suicide cases registered by the Legal Medicine Organization (Hasanzadeh and Rajaee 1984; Heidari 1997; Jamshidzadeh et al. 2004; Moradi and Khademi 2002; Yasami et al. 1998, 2002; Zarghami and Khalilian 2002).

For prevalence of lifetime suicide ideation findings of $12.7 \%$ (and 5.7\% at 12 months) are comparable withfindings obtained in similar investigations carried out in Sweden [8.6\% in 1986 and 12\% in 1996] (Renberg 2001), in the US (13.5\%) (Kessler et al. 1999), and Australia (10.4\%) (De Leo et al. 2005). Similarly, the results of the collaborative study conducted in Canada, France, Lebanon, New Zealand, Puerto Rico, Republic of Korea, Taiwan, the USA, and West Germany (Weissman et al. 1999), indicated the rate of lifetime prevalence of suicide ideation varied between 2 and 18\%. A more ample range of variability was witnessed in the context of the WHO/SUPRE-MISS study, where the eight participating countries showed variations in lifetime suicide ideation between 2.6 and 25.6\% (Fleischmann et al. 2005).

Rates of suicide plans obtained from the current study were higher than the prevalence data recorded in the US (3.9\%, lifetime) (Kessler et al. 1999) and Australia (4.4\%, lifetime) (De Leo et al. 2005). Within the WHO/SUPREMISS study, the range of variability for suicide plans among countries was $1.1-15.6 \%$. 
and the American (4.6\% for lifetime) (Kessler et al. 1999). In the study of Weissman et al. (1999), suicide attempt data varied between 0.7 and $6.0 \%$, whilst in the WHO/SUPRE-MISS study, suicide attempts ranged between 0.4 and $4.2 \%$.

We could notfind any data derived from community surveys conducted in Middle East or other Islamic coun tries to use as a comparison. A recent investigation performed in Pakistan (Zakiullah et al. 2008) was based on hospital-treated suicide attempters, and cannot be used for direct comparison with our data. However, the Pakistani study concurs with the present one in highlighting an epidemiological dimension of non-fatal suicidal behaviors that is very similar to that of many western countries. A study conducted by Pritchard and Amanullah (2007) using the latest WHO mortality data, concluded that suicide among Islamic countries varied widely, and Other Violent Deaths (OVD), according to ICD-9 definition, could be a "repository for hiding culturally unacceptable suicides”.

Despite the recorded prevalence of suicide ideation and that of plans and attempts obtained from the present study, the rate of deaths in Iran due to suicide (with the possible exception of some western cities in the country) is remarkably lower (6 suicides per 100,000 people) than in most European countries (on average, 12-40 suicides per 100,000 people, excluding Mediterranean countries), Australia and the US. Similarities and differences in rates of suicide would provide an opportunity for researchers to explore risks and protective factors in a cross-cultural perspective.

Lower rates of both fatal and non-fatal suicidal behavior in Iran could be related to factors such as sample bias, inadequate registration and reporting, stigma, religious beliefs and recall bias, discussed further below. Sample bias is possibly the most appreciable limitation of this study. Due to organizational reasons, the most common hours to conduct interviews were in the morning; however, men were normally less present at home at that time. In addition, most of the interviewers were female subjects and, due to the local culture, those men actually at home may have delegated the filling in of the questionnaires to female members of the family. As a possible result of these facts, in this study, the age group 15-29 comprised 55\% of subjects, whereas in the general population it constitutes $47 \%$ of subjects. On the other hand, the percentages of females and males in the present study were 64.8 and $35.2 \%$, but in the general population they are 48.7 and $51.3 \%$, respectively. It could be hypothesized that the higher proportion of females and young subjects in the study may have facially inflated rates for suicidal ideation and behavior. However, it is worth noticing that the Iranian sample used in occasion of the WHO/SUPREMISS study, reflective for age and gender of the exact distribution of the general population of the country (Bertolote et al. 2005), was obtained through consecutive inclusion of subjects in each age and gender category, with a process involving a big number of refusals; in any case, that survey has produced very similar results to the present one, showing an even higher percentage of suicide attempts (4.2 vs. $3.3 \%$ of this investigation). Therefore, despite the existence of sampling bias, it seems that the results of this study could be credibly generalized to the population of the Karaj city. Another factor affecting lower rates of both fatal and non-fatal suicidal behavior in developing countries such as Iran is a lack of an accurate and reliable system of registration and reporting, which may artificially lower the rate of suicide compared to non-fatal suicidal behavior data obtained from a community survey. The stigma embedded with mental disorders, and considering suicide as a mortal sin, may cause people not to report to oficials. Religious beliefs may act as a strong protective factor against suicide, but may act less strongly against suicide ideation, plans and behaviors. This could partly assist in explaining the apparent discrepancy between relatively high percentages of non-fatal suicidal behaviors and relatively low rates of suicide. Finally, a recall bias (common in this type of investigations) could potentially have affected the prevalence of lifetime ideation and behavior, by reducing their total numbers.

The higher rates of suicide recorded in some western cities of Iran could be explained by the more frequent use of self-burning as a common method of suicide attempt. It is possible that, as described in China by Phillips et al. (2002), a number of impulsive attempts, particularly using the method of self-immolation (Ahmadi and Yllerstad 2007; Ahmadi et al. 2008), could end in death due to the high lethality of the means employed. However, more research is needed to confirm this hypothesis.

\section{Conclusions}

Suicidal ideation and behaviors are not uncommon in Iran. As a result of this study, their epidemiological dimension appears to be aligned with that of several western countries, where suicide phenomena are presently being contrasted through the implementation of national suicide prevention strategies. Where a noticeable discrepancy exists, it is in the rate of suicide completion, which in Iran seems to be much lower than in western countries. It is unclear whether this evidence mirrors reality or represents instead an artifact (due to many different factors); surely this topic would deserve spefiic attention. In any cas e, the study here presented has provided a picture of non-fatal suicidality in the community that calls for quick interventions from health authorities and could be useful in the planning of a national suicide prevention strategy. 
research. This initiative was funded partly by the Mental Health Research Center (grant no. 2128) and partly by the Iran University of Medical Sciences, Research Department (grant no. 4118). We are also grateful to Mrs. Wendy Iverson, for her careful revision of the manuscript.

\section{References}

Agoub, M., Moussaoui, D., \& Kadri, N. (2006). Assessment of suicidality in a Moroccan metropolitan area. Journal of Affective Disorders, 90, $223-226$. doi:10.1016/j.jad.2005.09.014.

Ahmadi, A., \& Yllerstad, B. (2007). Prevention of self-immolation by community-based intervention. Burns, 33, 1032-1040. doi: 10.1016/j.burns.2006.11.015.

Ahmadi, A., Mohammadi, R., Slavrinos, D., Almasi, A., \& Schwebel, D. (2008). Self-immolation in Iran. Journal of Burn Care \& Research (Official Publication of the American Burn Association), $29,451-460$.

Bertolote, J. M., Fleischmann, A., De Leo, D., Bolhari, J., Botega, N., De Silva, D., et al. (2005). Suicide attempts, plans, and ideation in culturally diverse sites: The WHO SUPRE-MISS. Psychological Medicine, 35, 1457-1465. doi:10.1017/S003329170500 5404.

Bolhari, J., Hajian Motlagh, N., Asadi, A., \& Hasanzadeh, M. (2002). The prevalence of suicide and suicide attempt in Savojbolagh Iran. Final report of a research study. Tehran: Iran University of Medical Sciences.

De Leo, D., Cerin, E., Spathonis, K., \& Burgis, S. H. (2005). Lifetime risk of suicide ideation and attempts in an Auatrialian community: Prevalence, suicidal process, and help-seeking behaviour. Journal of Affective Disorders, 86, 215-224. doi:10.1016/j.jad. 2005.02.001.

Fleischmann, A., Bertolote, J. M., De Leo, D., Botega, N., Phillips, M., Sisask, M., et al. (2005). Characteristics of attempted suicides seen in emergency-care settings of general hospitals in eight low and middle income countries. Psychological Medicine, 35, 1467-1474. doi:10.1017/S0033291705005416.

Hasanzadeh, M., \& Rajaee, A. (1984). Study of 200 cases with suicide. Journal of Esfahan Department of Medicine (Farsi), 22, 47-59.

Heidari, P. A. (1997). The study of Psycho-social Factors and Epidemiological Characteristics of the people who Attempted Suicide in Hamadan, Iran. Andisheh-va-raftar, 3, 19-31. (Farsi).

Jamshidzadeh, F. L., Rafeiy, H., Yasami, M. T., Rahimi, A. S., \& Aazam, K. (2004). The procedure of Changes in Ilam province related to suicide practice. Social Welfare Quarterly, 3, 325-345. (Farsi).

Kessler, R. C., Borges, G., \& Walters, E. E. (1999). Prevalence of and risk factors for lifetime suicide attempts in the National comorbidity survey. Archives of General Psychiatry, 56, 617-

626. doi:10.1001/archpsyc.56.7.617.

Liu, Z., Tein, J.-U., Zhao, Z., \& Sandler, I. N. (2005). Suicidality and correlates among rural adolescents of China. The Journal of Adolescent Health, 37, 443-451. doi:10.1016/j.jadohealth.2004.

08.027 .

Moosavi, F., Shahmohammadi, D., \& Kafashi, A. (2000). The prevalence of suicide in rural area. Andishe-va-raftar, 3, 4-10. (Farsi).

Moradi, S., \& Khademi, A. (2002). Evaluation of suicides resulting in death in Iran, comparing with the World rates. Law Medicine Journal, 8, 16-21. Farsi.

Naghavi, M., \& Akabari, M. E. (2002). The epidemiology of injuries in Islamic Republic of Iran. Tehran: Iran Ministry of Health, Undersecretary of Health, Fekrat Publication.

Nojomi, M., Malakouti, S. K., Bolhari, J., \& Poshtmashhadi, M. (2007). A predictor model for suicide attempt: Evidence from a population-based study. Archives of Iranian Medicine, 10, 452-

458.

Phillips, M. R., Yang, G., Zhang, Y., Wang, L., Ji, H., \& Zhou, M. (2002). Risk factors for suicide in China: A national case-control psychological autopsy study. Lancet, 360, 1728-1736. doi: 10.1016/S0140-6736(02)11681-3.

Pritchard, C., \& Amanullah, S. (2007). An analysis of suicide and undetermined deaths in 17 predominantly Islamic countries contrasted with the UK. Psychological Medicine, 37, 421-430. doi:10.1017/S0033291706009159.

Renberg, E. S. (2001). Self-reported life-wearing, death-wishes, suicidal ideation, suicidal plans and suicide attempts in general population surveys in the north of Sweden 1986-1996. Social Psychiatry and Psychiatric Epidemiology, 36, 429-436. doi: 10.1007/s001270170020.

Rey, C., Michaud, P. A., Narring, F., \& Ferron, C. (1997). Suicide ideation and behavior in Swiss adolescents: A concern for the physicians. Archives de Pediatrie, 4, 784-792. doi:10.1016/ S0929-693X(97)83424-0.

Weissman, M. M., Bland, R. C., Canino, G. J., Greenwald, S., Hwu,

H. G., Joyce, P. R., et al. (1999). Prevalence of suicide ideation and suicide attempts in nine countries. Psychological Medicine, 29, 9-17.

World Health Organization, Regional Office for Europe (2005) Mental Health: Facing with challenges and solutions. Report from the WHO European ministerial conference.

Yasami, M. T., Sanei, N., Malekpour, R., Honarmand, A. R., \& Mirshekari, R. (1998). Epidemiological study of attempted suicide in Kerman, Iran. Andisheh-va-raftar, 3, 15-35. (Farsi).

Yasami, M. T., Sabahi, A. R., \& Mir Hashemi, M. (2002). Epidemiological survey of suicide through the Forensic Medical Center in the Province of Kerman, Iran. Andisheh-va-raftar, 7, 4-12. (Farsi).

Zakiullah, N., Saleem, S., Sadiq, S., Sani, N., Shapurwala, M., Shamim, A., et al. (2008). Deliberate self-harm. Characteristics of patients presenting to a tertiary care hospital in Karachi, Pakistan. Crisis, 29, 32-37. doi:10.1027/0227-5910.29.1.32.

Zarghami, M., \& Khalilian, A. (2002). Deliberate self-burning in Mazandaran, Iran. Burns, 28, 115-119. doi:10.1016/S0305-4179 (01)00092-4. 
Table 1 Demographic features of the study sample in proportion of suicide thought, planning and attempt in life time

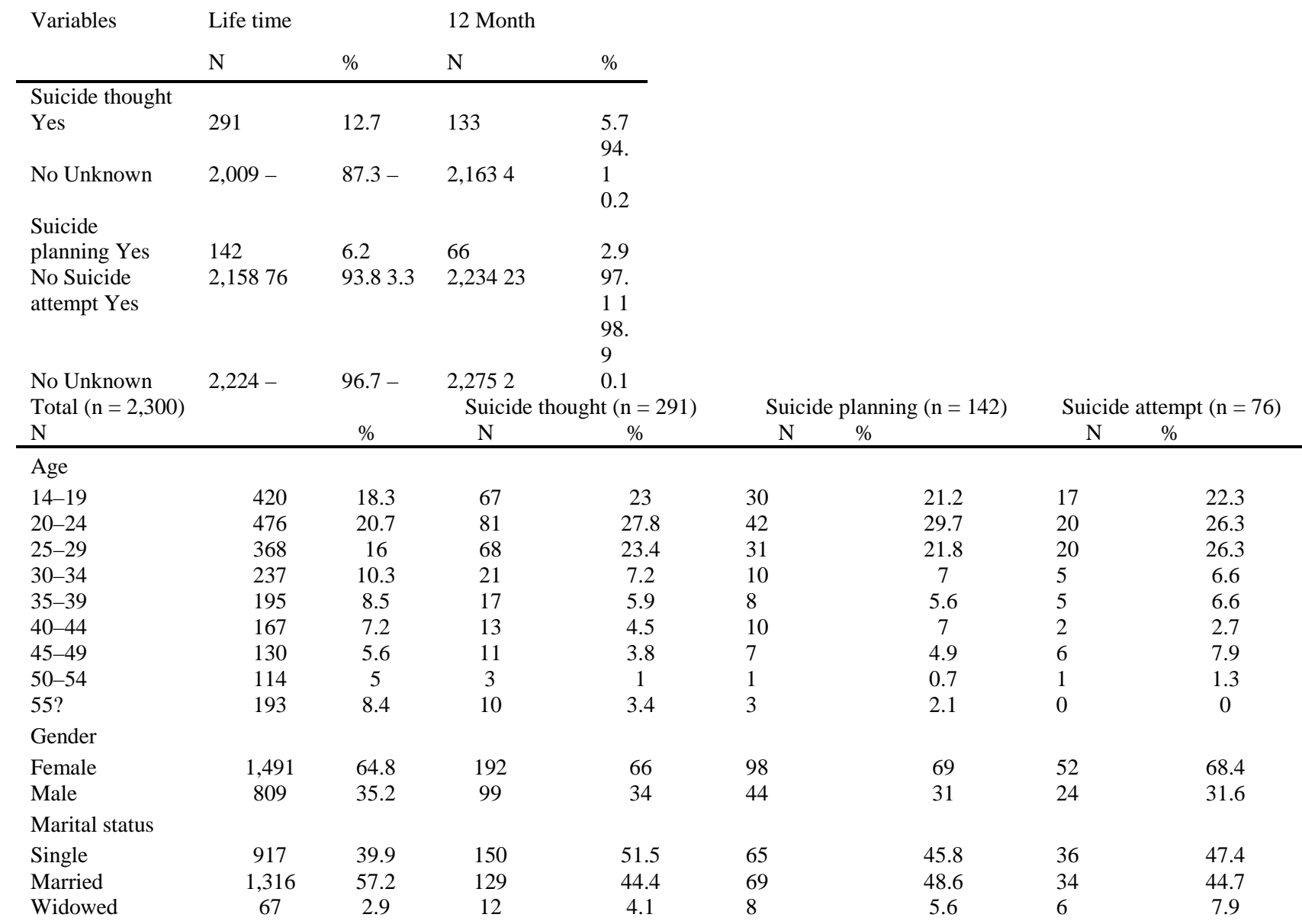

Missed or non-applicable data were not appeared in the table

Table 2 The frequency of suicide thought, planning and attempt in life time and 12 months

\begin{tabular}{|c|c|c|c|c|}
\hline \multirow[t]{2}{*}{ Variables } & \multicolumn{2}{|l|}{ Life time } & \multicolumn{2}{|c|}{12 Month } \\
\hline & $\mathrm{N}$ & $\%$ & $\mathrm{~N}$ & $\%$ \\
\hline \multicolumn{5}{|l|}{ Suicide thought } \\
\hline Yes & 291 & 12.7 & 133 & $\begin{array}{l}5.7 \\
94 .\end{array}$ \\
\hline No Unknown & $2,009-$ & $87.3-$ & 2,1634 & $\begin{array}{l}1 \\
0.2\end{array}$ \\
\hline $\begin{array}{l}\text { Suicide } \\
\text { planning Yes }\end{array}$ & 142 & 6.2 & 66 & 2.9 \\
\hline $\begin{array}{l}\text { No Suicide } \\
\text { attempt Yes }\end{array}$ & 2,15876 & 93.83 .3 & 2,23423 & $\begin{array}{l}97 . \\
11 \\
98 . \\
9\end{array}$ \\
\hline $\begin{array}{l}\text { No Unknown } \\
\text { Total }(n=2,300)\end{array}$ & $2,224-$ & $96.7-$ & $\begin{array}{l}\text { 2,275 } 2 \\
\text { Suicide }\end{array}$ & $\begin{array}{l}0.1 \\
\text { ht }(\mathrm{n}=291)\end{array}$ \\
\hline
\end{tabular}

